\title{
LITERATURE SURVEY ON SMART SURVEILLANCE SYSTEM
}

\author{
Mayuri Waghmare \\ Department of Computer Science \\ Bharti Vidyapeeth College Of Engineering, \\ Navimumbai, Maharashtra, India \\ Pranalee Shirsat \\ Department of Computer Science \\ Bharti Vidyapeeth College Of Engineering, \\ Navimumbai, Maharashtra, India
}

\begin{abstract}
The smart surveillance system defines an approach to identify and recognize human faces from the surveillance videos. It is very tedious to find particular person within a video. This system gives a quick and efficient method to find the presence of a person within a surveillance video.
\end{abstract}

Keywords- Face Detection, Face Recognition, Surveillance, Surveillance Video.

\section{INTRODUCTION}

This paper describes literature survey on "Smart Surveillance System". Surveillance cameras are widely used record videos of its surroundings in order to provide security in every place which demands security. If we wish to find the presence of a particular person within the surveillance video, we have to watch the complete video. This is a very tedious task. Sometimes it may even happen that the target person may go unnoticed in the video. This paper describes an application named "Smart Surveillance System". Surveillance cameras are widely used record videos of its surroundings in order to provide security in every place which demands security. If we wish to find the presence of a particular person within the surveillance video, we have to watch the complete video. This is a very tedious task. Sometimes it may even happen that the target person may go unnoticed in the video. To overcome these problems we have proposed a system named "A Smart Surveillance System", which uses machine learning approach to detect and recognize the target person in the video.

The rest of the paper is organized as follows. Proposed smart surveillance system is explained in section II. Various existing methods are studied and concluded as literature survey and presented in section III. Challenges are given in section IV. Concluding remarks are given in section in V.

\author{
Deepak Dhadve \\ Department of Computer Science \\ Bharti Vidyapeeth College Of Engineering \\ Navimumbai, Maharashtra, India \\ B.W.Balkhande \\ Department of Computer Science \\ Bharti Vidyapeeth College Of Engineering, \\ Navimumbai, Maharashtra, India
}

\section{PROPOSED SYSTEM}

A smart surveillance system is a desktop application which detects the presence of any person in a surveillance video. This desktop application uses machine learning to perform the task. Various machine learning techniques like face recognition and face detection can be used to find particular faces in the video.

In the traditional video surveillance systems, if we want to find the presence of a particular person in the video, an employee had to be assigned to watch the video surveillance footage in order to find the person. This task thus becomes very tedious and time consuming. Along with this, the identification task is done by humans and humans are prone to make mistakes. It may happen that the target person has appeared in the video but has been overlooked by the person assigned to perform the task.

In order to overcomes these problems, we have proposed the idea of this application, "The Smart Surveillance System'. This system will automatically identify the target person in the surveillance video. Hence, there is no need of a person to analyze the video manually. Furthermore, the computer has higher processing speed as compared to the humans which speeds up the process of identification and saves the time. As the application uses machine learning, the target person cannot be overlooked by the application even in a clustered surveillance video. This application can be used for various security measures. This software can be used to find any person in a video. Thus, if a person is found missing, then we can use this application to check if the missing person has been seen in a particular area at any time. This would help is finding the person easily and quickly. Similarly, it can also be used to find thieves or wanted criminals by just using the image of them. It can be used to check if a particular criminal was seen an area at any time. This application can be used to find any person in a crowd or cluttered video. 


\section{International Journal of Engineering Applied Sciences and Technology, 2020 Vol. 4, Issue 12, ISSN No. 2455-2143, Pages 170-173 \\ Published Online April 2020 in IJEAST (http://www.ijeast.com)}

Apart from the security field, this application can also be used efficiently in offices to check the working hours of employees. This application can be used to find the arrival and the leaving time of any employee in the office. On finding this, the application can find the time spent by the employee working.

\section{LITERATURE SURVEY}

In [1] earlier system has many problem such as such as high cost, low intelligence, weak security.S3C2440 microprocessor is used in this embedded video acquisition system, which combing with the Linux operating system. This paper has advantages such as higher intelligence, higher stability, and easy installation and disadvantage as it requires high cost and continues GSM network is required to send message, if the network is not available this project may not

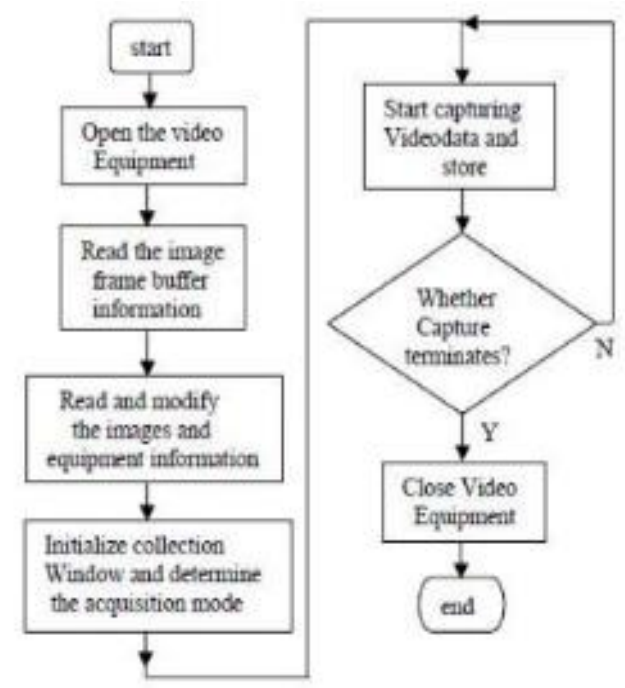

Fig. 1.Flowchart of V4L video capture

work properly. Below is the diagram showing the flowchart of Video4Linux that has been used in this paper. In [2] a query algorithm is introduced which will be used to establish the relationship between query and database video by bipartite graph, it construct a sub graph which matches the max size and it neglects the small one. Here two system are mainly used:

\section{- Video frame Detection}

- Video Similarity Search

Deep packet capture (DPC) is the act of capturing complete network packets crossing a network. Here we capture data packets in the scalable network using packet capturing tools. In [3] Network video capture system using friendly ARM9 board support package (BSP) S3C2440 is presented. This application takes video, shares video in networked systems and also alerts the controlling person with short message service alarm. This system is advantageous in providing low cost and high effective intelligent monitoring system like in elevators, home security systems etc. with low power consumption. Here RT Linux is used i.e. Real time operating system and also it can be wired or wireless internet access this could be its advantage. It alerts the person through the Short message service (SMS).

The [4] paper deals with the integrated server and MPEG video. The integrated server means that a specific server is located in the centre of the surveillance system this can get the video information from the network camera and then compress that video and store it for future use. It can also receive request from the clients like monitoring, controlling etc. The method used in this paper is the open PLANET technology that has been developed by Shikoku Electric Power Company Ltd it has some excellent characteristics, one of which is to transmit digital information from node to node through electric power line. Its advantages include low maintenance, cost effective and mobile operation i.e. it can operated from anywhere and also on the mobile phones, desktop as per user convenience.

The [5] paper is about the development of ov511 USB camera in Linux operation system. ov511 USB camera is used here for capturing of video and embedded Linux platform which is based on S3C2440 micro controls chip. The processing of the captured video is based on video4linux. Below shows the diagram the Structure of video capturing system. The advantages of this paper will be Rapid video acquisition, Real time transmission well, Stable performance and lower cost.

In paper [6] the video captures the motion is detected and SMS is sent. Video4Linux is used here to get the video data then is it transferred to the web server and displayed on the client side. The advantages of includes fewer modules, low cost, higher intelligence, higher stability and higher security. It utilizes the AT commands to give the SMS alarm and can realize remote monitoring. In this when the object is not there the screen shows green color and when the object come and the screen is interrupts with any different object it shows red color on the screen.

\section{CHALLENGE}

Many challenges are faced in order to get the expected outcomes. Few of the challenges faced are as follows:

\section{- Storage}

A video may contain many faces. Especially in a crowded area, where many people are present at the same time. In order to make the application more accurate, every face in the video should be extracted so that no face is missed by the application. In addition to this the size of the videos may vary and some video may be large in size. Large video contain large number of frames from which faces are extracted. Due to this a large number of faces are to be saved. Hence, the size of the storage is huge. 


\section{International Journal of Engineering Applied Sciences and Technology, 2020 Vol. 4, Issue 12, ISSN No. 2455-2143, Pages 170-173 \\ Published Online April 2020 in IJEAST (http://www.ijeast.com)}

- Faces in background

A video frame may contain faces in various positions. Some in the front of the scene which appear clear and distinct while some in the back which may not appear as clear and distinct as the other faces. The features of the faces may not be clear. Hence it becomes difficult to identify the faces present at the back in any given video.

\section{- Partial faces}

In a particular video frame, sometimes it may happen that a person do not enter the video frame completely. The face of the person may appear partially in the video. In such times it becomes difficult to detect the face in the video. It may also happen that a person is not facing the camera but, is facing sideways. In such cases, it becomes difficult to identify a person to be the target person only by seeing its side face. Detecting a face leaned sideways and then comparing it to the target face is also difficult as the orientation of the face may change the values of the eigen vector.

\section{- Similar looking people}

Often it may happen that two people look similar to each other upto some extend. Thus, one person can easily be confused with another and so can be their faces. It becomes important that similar looking people can be distinguished with each other to avoid the confusion of identifying the wrong person as the target person.

\section{- No training set}

As the image of the target person is provided by the user in real time, no training set is available to tain the model for identifying the target person. We match the face of the target person directly to the faces extracted from the videos. It would also be inconvenient to ask the user for multiple images of the target person who is to be found in the videos. Thus, it becomes difficult to identify the faces without a training set.

\section{REFERENCE}

[1] Volume 3, Issue 3, March 2013 ISSN: 2277 128X

International Journal of Advanced Research in Computer Science and Software Engineering Research Paper Sixth International Conference on Natural Computation (ICNC 2010) "Network Video Capture and Short Message Service Alarm System Design Based on Embedded Linux" Duanchun ZHOU Guangxing TAN.

[2] International Journal of Innovative Research in Computer and Communication Engineering (An ISO 3297: 2007 Certified Organization)Vol.1, Issue 8, October 2013 "IMPLEMENTATION OF EMBEDDED VIDEO RETRIEVE SYSTEM BASED ON ARM9" V. Jyothi, Prof. M.Srinivas Rao,K.Hanuja
[3] "ARM9 Based Real Time Embedded Network Video Capture And SMS Alerting system"

M.L.V.N.B.S.Kumar, Dr.N.S.Murthi Sarma, Ch.Sridevi, A.Pravin ECE Department, BVC Engineering College Odalarevu(2012) F. Gonzalez and J. Hernandez, " A tutorial on Digital Watermarking ", In IEEE annual Carnahan conference on security technology, Spain, 1999.

[4] International Journal of Innovative Research in Computer and Communication Engineering (An ISO

3297: 2007 Certified Organization)Vol.1, Issue 8, October 2013 "IMPLEMENTATION OF EMBEDDED VIDEO RETRIEVE SYSTEM BASED ON ARM9" V.

[5] IOSR Journal of Engineering (IOSRJEN) e-ISSN: 22503021, p-ISSN: 2278-8719 Vol. 3, Issue 7 (July. 2013), ||V6||PP 14-1814 | P a g e "Embedded Real Time

Video Monitoring System using Arm" by Kavitha Mamindla, Dr.V.Padmaja, CH.NagaDeepa.

[6] "VIDEO MONITORING AND MOTION DETECTION SYSTEM BASED ON ARM-LINUX PLATFORM AND HTTP PROTOCOL WITH SMS CAPABILITY" *Nava Jeevan Raju, O. and Praveen, P.(2014).

[7] Liton Chandra Paul1, Abdulla Al Sumam, "Face Recognition Using Principal Component Analysis Method" in International Journal of Advanced Research in Computer Engineering \& Technology (IJARCET) Volume 1, Issue 9, November 2012

[8] Hui Shuai, Qingshan Liu, Kaihua Zhang, Jing Yang, Jiankang Deng, "Cascaded Regional Spatio-Temporal Feature-Routing Networks for Video Object Detection",in Wadsworth, 1993, pp.123-135.

[9] Himani S. Parekh1, Darshak G. Thakore 2, Udesang K. Jaliya, "A Survey on Object Detection and Tracking Methods" in International Journal of Innovative Research in Computer and Communication EngineeringVol. 2, Issue 2, February 2014.

[10] Conference on Environmental Science and Information Application Technology-"A Wireless Video Surveillance System based on 3G Network" by Wang Liwei Yan Shi $\mathrm{Xu}$ Yiqiu.

[11] J. N. K. Liu, M. Wang, and B. Feng, iBotGuard: an Internet-based intelligent robot security system using invariant face recognition against intruder, IEEE Transactions on SystemsMan And Cybernetics Part CApplications And Reviews,

[12] K. Balci and V. Atalay, PCA for Gender Estimation: Which Eigenvectors Contribute? in Proceedings of Sixteenth International Conference on Pattern Recognition, Vol.3. Quebec City, Canada, 2002, pp. 363366. 


\section{ACKNOWLEDGMENT}

This paper was possible because of the able guidance of our professor Prof. B.W.Balkhande. The authors wish to express their sincere gratitude for providing the idea, which indeed helped us in doing a lot of Research and we came to know about many new things. We would also like to thanks others who helped us to fulfill this paper. Would also extend our heartfelt acknowledge to our parents for encouraging us. 